\title{
Are There Any Experimental Perfusion Data that Preferentially Support the Dispersion and Parallel-Tube Models over the Well-Stirred Model of Organ Elimination?
}

\author{
Jasleen K. Sodhi, Hong-Jaan Wang, and 느eslie Z. Benet
}

Department of Bioengineering and Therapeutic Sciences, Schools of Pharmacy and Medicine, University of California San Francisco, San Francisco, California (J.K.S., L.Z.B.) and School of Pharmacy, National Defense Medical Center, Taipei, Taiwan (H.J.W.)

Received January 13, 2020; accepted March 31, 2020

\section{ABSTRACT}

In reviewing previously published isolated perfused rat liver studies, we find no experimental data for high-clearance metabolized drugs that reasonably or unambiguously support preference for the dispersion and parallel-tube models versus the well-stirred model of organ elimination when only entering and exiting drug concentrations are available. It is likely that the investigators cited here may have been influenced by: 1) the unphysiologic aspects of the wellstirred model, which may have led them to undervalue the studies that directly test the various hepatic disposition models for highclearance drugs (for which model differences are the greatest); 2) experimental assumptions made in the last century, which are no longer valid today, related to the predictability of in vivo outcomes from in vitro measures of drug elimination and the influence of albumin in hepatic drug uptake; and 3) a lack of critical review of previously reported experimental studies, resulting in inappropriate interpretation of the available experimental data. The number of papers investigating the theoretical aspects of the dispersion, parallel-tube, and well-stirred models of hepatic elimination greatly outnumber the papers that actually examine the experimental evidence available to substantiate these models. When all experimental studies that measure organ elimination using entering and exiting drug concentrations at steady state are critically reviewed, the simple but unphysiologic well-stirred model is the only model that can describe all trustworthy published available data.

\section{SIGNIFICANCE STATEMENT}

Although the dispersion model of hepatic elimination more adequately reflects physiologic reality, there are no convincing experimental data that unambiguously favor this model. The well-stirred model can describe all well-designed perfusion studies with highclearance drugs and nondrug substrates, but the field has not recognized this because of hesitation to accept a nonphysiologic model and flawed attempts to utilize in vitro-in vivo extrapolation approaches.

\section{Introduction}

Forty-eight years ago, Rowland (1972) defined steady-state organ clearance [here, hepatic clearance $\left.\left(C L_{H}\right)\right]$ as the fraction of the entering drug blood concentration $\left(C_{i n}\right)$ that is eliminated by the organ multiplied by organ hepatic blood flow $\left(Q_{H}\right)$ with the ratio of concentration terms designated as the extraction ratio $(E R)$.

$$
C L_{H}=Q_{H} \cdot \frac{C_{\text {in }}-C_{\text {out }}}{C_{\text {in }}}=Q_{H} \cdot\left(1-\frac{C_{\text {out }}}{C_{\text {in }}}\right)=Q_{H} \cdot E R
$$

This work was supported in part by a Mary Ann Koda-Kimble Seed Award for Innovation. J.K.S. was supported in part by an American Foundation for Pharmaceutical Education Predoctoral Fellowship, the National Institutes of General Medical Sciences [Grant R25 GM56847], and a Louis Zeh Fellowship. L.Z.B. is a member of the University of California San Francisco (UCSF) Liver Center, which is supported by the National Institutes of Health [Grant P30 DK026743].

https://doi.org/10.1124/dmd.120.090530.
This simple but useful relationship allowed for clearance measurements based only on knowledge of entering and exiting concentrations and organ blood flow. In 2018, Benet et al. (2018) maintained that eq. 1 was only consistent with the well-stirred model of hepatic elimination, since the amount lost at steady state, $Q_{H} \cdot\left(C_{i n}-C_{\text {out }}\right)$, was divided by the drug concentration entering the liver $\left(C_{i n}\right)$ to obtain $C L_{H}$, and no other concentrations within the liver were considered in the clearance determination. These are solely characteristics of the well-stirred model. In a commentary accompanying that paper, Rowland and Pang (2018) wrote that eq. 1 is model-independent and "simply express[es] proportionality between observed rate of elimination and a reference concentration," and as they had earlier indicated (Pang and Rowland, 1977), that "by definition" organ clearance is given by eq. 1 .

In the present manuscript, we do not further discuss the theoretical differences of Benet et al. (2018) versus Rowland and Pang (2018) with respect to eq. 1; rather, we objectively review and critically evaluate the experimental data available when eq. 1 is used to calculate organ clearance. If there is truth to the assertion that eq. 1 is modelindependent, there should be experimental data supporting preference for the dispersion or parallel-tube models versus the well-stirred model

ABBREVIATIONS: $C_{i n}$, entering drug blood concentration; $C L_{H}$, hepatic clearance; $C L_{\text {int }}$, intrinsic clearance; $C_{\text {out }}$, exiting drug blood concentration; $E R$, extraction ratio; $F$, hepatic availability; $f_{u}$, fraction unbound; IPRL, isolated perfused rat liver; IVIVE, in vitro-in vivo extrapolation; $Q_{H}$, hepatic blood flow; $R_{N}$, efficiency number. 
when eq. 1 is used to calculate organ clearance. There are numerous papers related to the theoretical basis of alternate models of hepatic elimination (which we agree are more physiologically relevant than the well-stirred model) and even more papers by hundreds of authors throughout the field in which such models are used, including by widely employed physiologically based pharmacokinetic (PBPK) programs. However, there are very few experimental papers that directly test the differences between the theoretical organ disposition models as we review here. Let us be clear. We agree that the well-stirred model (also called the venous-equilibration model) is unphysiologic. We agree that there is zonal distribution of the metabolic activity of enzymatic processes within the liver. We agree that there is dispersion within the liver that is neither zero nor infinite. The purpose of this paper is to examine for the first time all of the experimental data when only entering and exiting concentrations for an organ of elimination are available with respect to which model is consistent with the eq. 1 definition of hepatic elimination.

\section{Methods}

Literature Search. Previously published isolated perfused rat liver (IPRL) studies were identified from the literature because it is possible to directly distinguish the models of hepatic elimination with such isolated organ studies and because such IPRL studies were commonly used and cited by the field as support of one hepatic disposition model versus another. Since the well-stirred model, dispersion model, and paralleltube model quantitatively predict similar clearance values for low and moderately extracted drugs, analysis focused only on high extractionratio substrates $(E R>0.7)$ in which the models could maximally be discriminated from one another. The literature search resulted in identification of only four publications that performed IPRL studies for high-clearance drugs [lidocaine (Pang and Rowland, 1977; Ahmad et al., 1983), meperidine (Ahmad et al., 1983), and propranolol (Jones et al., 1984, 1985)] in which model differentiation was possible. Four additional studies were identified for two high-clearance nondrug substances [galactose (Keiding and Chiarantini, 1978) and taurocholate (Smallwood et al., 1988; Ching et al., 1989; Roberts et al., 1990)] and five studies for which the low-clearance drugs, diazepam and diclofenac, were manipulated to behave like a high-clearance drug by altering protein binding (Rowland et al., 1984; Ching et al., 1989; Diaz-Garcia et al., 1992; Hussein et al., 1993; Wang and Benet, 2019). All discussed publications are listed in Table 1 . These studies were critically examined with respect to the degree of discrepancy that the experimental data had with predictions from each hepatic disposition model with the purpose of potentially identifying experimental data that cannot be described by the well-stirred model.

In evaluating the validity of results of IPRL studies, it is important to ensure that the viability of the IPRL is maintained throughout the experimentation period. Key points to consider include 1) length of perfusion times, with preference for shorter duration times, 2) ensuring elimination follows first-order kinetics at concentrations tested, 3) that flows should be optimized as to not damage the liver at very high flow rates and ensure the vasculature is fully perfused, which may be an issue for flow rates that are too low, and 4) adequate oxygenation of the system. For the purposes of model discrimination, the selected drugs should be high $E R$ and perfusion rate-limited.

Model Discrimination. Investigators conducted IPRL studies with known $C_{\text {in }}$ values and measured $C_{\text {out }}$ values under different experimental conditions that alter experimental flow or protein binding [fraction unbound $\left(f_{u}\right)$ ]. Experimental results were reported as $E R$, availability $\left(F=\frac{C_{\text {out }}}{C_{\text {in }}}\right)$, or the ratio of observed $C_{\text {out }}$ values under different experimental conditions. Investigators then compared these observed outcome measurements with the predicted value expected for the wellstirred model versus the values expected for the alternate parallel-tube and dispersion models. In all of these studies, clearance was calculated by eq. 1 under the assumption that eq. 1 is model-independent. It should be noted that if eq. 1 is not model-independent, then the expected values for the parallel-tube and dispersion models reported by these investigators would be incorrect. However, here we accept these comparisons and the model-independent assumption of eq. 1 to objectively evaluate the available experimental data under the same assumptions made by the investigators.

In Vitro-In Vivo Extrapolation Approach. An alternate indirect approach to test model discrimination was previously proposed by Roberts and Rowland (1986) and further presented by Iwatsubo et al. (1996) in which in vitro measures of intrinsic clearance $\left(C L_{\text {int }}\right)$ were scaled up to predictions of in vivo $C L_{\text {int }}$ after physiologically based in vitro-in vivo extrapolation (IVIVE) techniques for a number of drugs with published IPRL data. These IVIVE-predicted in vivo $C L_{\text {int }}$ values were then used to calculate an efficiency number $\left(R_{N}\right)\left(R_{N}=f_{u} \cdot C L_{\text {int }}\right.$ / $Q_{H}$ ) based on IPRL experimental conditions and plotted against experimentally observed hepatic availability $(F)$, which was calculated using eq. 1 and the extraction-ratio/bioavailability relationship $(E R=$ $1-F)$. Finally, these values were compared with the hepatic availability values expected for the various hepatic disposition models.

\section{Results}

IPRL Studies of High-Clearance Drugs. The various hepatic disposition models diverge from one another as clearance value increases, therefore high-clearance (extraction ratio) compounds are the most appropriate for testing model discrimination. There are only four published IPRL studies that evaluate these models for high extraction-ratio drugs (Pang and Rowland, 1977; Ahmad et al., 1983; Jones et al., 1984, 1985). All four of those studies, including two from the Rowland laboratory, conclude that the data are consistent with the well-stirred model and not alternate hepatic clearance models.

In the first of the two Rowland publications, Pang and Rowland (1977) evaluated the effect of changing organ blood flow on the extraction ratio of lidocaine as depicted in Fig. 1. The title of the paper indicates the results: "Experimental evidence for acceptance of the wellstirred model over the parallel-tube model using lidocaine in the perfused rat liver in situ preparation." In the second Rowland publication, Ahmad et al. (1983) also evaluated the effect of changing blood flow on the extraction ratio of lidocaine and meperidine (pethidine), displaying figures that markedly differentiate the experimental outcome between the well-stirred model and the parallel-tube model for both drugs. The concluding sentence of the abstract of that paper states, "The experimental findings indicate that the well-stirred model more accurately predicts the elimination of highly cleared drugs with perturbation of flow than does the parallel tube model."

Two additional studies were published by Jones et al. (1984, 1985) that evaluated the effect of changing protein binding on the extraction ratio of propranolol. The advantage of altering protein binding (as opposed to flow) is that it is possible to vary binding over a much larger range than flow can be varied because of liver integrity issues resulting from flow rates that are too high or too low. In the Jones et al. (1984) study, a 2.7-fold change in propranolol protein binding was achieved when albumin concentration was varied. The following year Jones et al. (1985) reported an average 5.7-fold change in protein binding when $\alpha_{1^{-}}$ acid glycoprotein was the binding protein examined. In contrast, in the Rowland studies, flow was only able to be varied by 1.6-fold (Pang and Rowland, 1977) and 1.5-fold (Ahmad et al., 1983). Results for both Jones et al. studies show clear preference for the well-stirred model, and 
TABLE 1

Summary of IPRL studies for high-clearance substrates

\begin{tabular}{|c|c|c|c|}
\hline Test compound & Category & Condition Altered & Reference \\
\hline Lidocaine & Drug & $Q_{H}(1.6$-fold $)$ & Pang and Rowland, 1977 \\
\hline Lidocaine & Drug & $Q_{H}(1.5$-fold $)$ & Ahmad et al., 1983 \\
\hline Meperidine (pethidine) & Drug & $Q_{H}(1.5$-fold $)$ & Ahmad et al., 1983 \\
\hline Propranolol & Drug & $f_{u}(2.7$-fold $)$ & Jones et al., 1984 \\
\hline Propranolol & Drug & $f_{u}(5.7$-fold $)$ & Jones et al., 1985 \\
\hline Galactose & Nondrug substrate & $Q_{H}(1.4$ to 1.8 -fold $)$ & Keiding and Chiarantini, 1978 \\
\hline Taurocholate & Nondrug substrate & $\tilde{f}_{u}$ (single-pass: 11.1 -fold $)$ & Smallwood et al., 1988 \\
\hline Taurocholate & Nondrug substrate & $f_{u}$ (recirculating: 18 -fold) & Smallwood et al., 1988 \\
\hline Taurocholate & Nondrug substance & $f_{u}(14.6$-fold $)$ & Ching et al., 1989 \\
\hline Taurocholate & Nondrug substrate & $f_{u}$ (7.4-fold) & Roberts et al., 1990 \\
\hline Taurocholate & Nondrug substrate & $Q_{H}(3.7$-fold $)$ & Roberts et al., 1990 \\
\hline Diazepam $^{a}$ & Drug & $f_{u}$ (13.2-fold) & Ching et al., 1989 \\
\hline Diazepam $^{a}$ & Drug & $f_{u}(1.3$-fold $)$ & Rowland et al., 1984 \\
\hline Diazepam $^{a}$ & Drug & $f_{u}(2.7$-fold $)$ & Diaz-Garcia et al., 1992 \\
\hline Diazepam $^{a}$ & Drug & $Q_{H}(2.0$-fold $)$ & Diaz-Garcia et al., 1992 \\
\hline Diazepam $^{a}$ & Drug & $f_{u}(1.4$-fold $)$ & Wang and Benet, 2019 \\
\hline Diclofenac $^{a}$ & Drug & $f_{u}(333$-fold $)$ & Hussein et al., 1993 \\
\hline
\end{tabular}

${ }^{a}$ Low-clearance drug manipulated to be high-clearance in absence of plasma proteins.

at the beginning of the concluding paragraph of the Jones et al. (1984) paper, they state, "Although there may be no simple anatomical explanation for the applicability of one or other model, it is clear that the venous equilibration model precisely describes the hepatic elimination of propranolol. Operationally, the liver is behaving as a well mixed compartment, however 'unphysiological' this may seem." In the first sentence of the concluding paragraph of Jones et al. (1985), they warn that "the a priori thinking which rules out the venous equilibrium model on the grounds of physiological 'irrelevance' deserves careful reappraisal." In the final sentence, Jones et al. (1985) quote Cobelli et al. (1984): "it would seem unjustified to contravene the "principle of parsimony' by invoking a more complex model when the simpler model will do."

The four studies above are the only IPRL papers published in the literature for high-clearance drugs, and they are all consistent with the well-stirred model as acknowledged by the authors.

IPRL Studies of Nondrug Substrates. Nondrug substances have also been studied in IPRL experiments. A frequently cited study is that of Keiding and Chiarantini (1978), which examined galactose elimination in recirculating rat liver perfusions versus the single-pass perfusions used in the studies discussed above. Although this paper, which

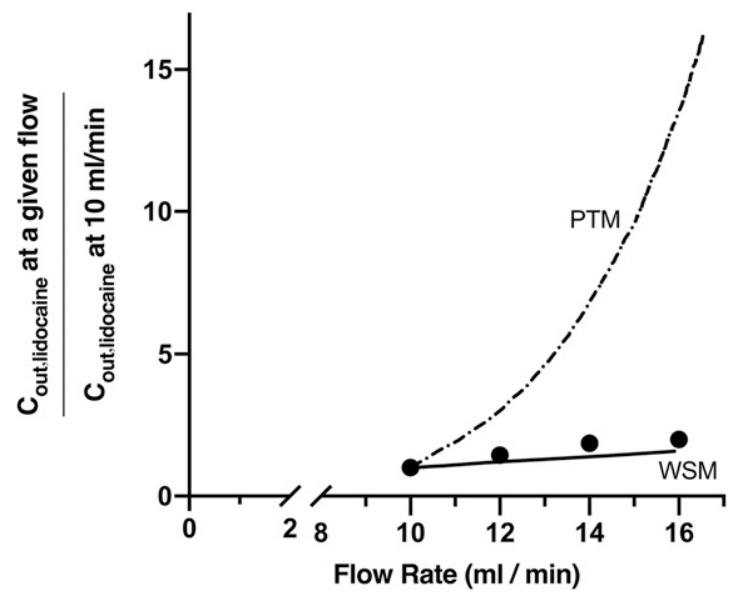

Fig. 1. Experimental lidocaine IPRL results and models of hepatic elimination from Pang and Rowland (1977). The well-stirred model (WSM) appears as a solid line and the parallel-tube model (PTM) appears as a dashed line. concludes that sinusoidal perfusion (the parallel-tube model) is consistent with the experimental data, is frequently cited, it appears the field has accepted the conclusion without examination of the experimental data. Galactose had been previously demonstrated to be a high-clearance substrate by Goresky et al. (1973), who report that "the extraction is almost complete, i.e., the hepatic venous blood is almost completely cleared of galactose" at the galactose concentrations tested by Keiding and Chiarantini (1978). However, the experimental results of the Keiding and Chiarantini (1978) study are not consistent with galactose being a high-clearance substance as would be expected for galactose. At flow rates of 10 to $11 \mathrm{ml} / \mathrm{min}$, the lowest $C_{\text {ou }} / C_{\text {in }}$ values of the 10 experiments reported are 0.32 and 0.34 (experiment 9), corresponding to $E R$ values of 0.68 and 0.66 . However, the average $E R$ value for the 20 measurements in the 10 experiments was $0.46 \pm 0.11$ (S.D.). For the 6 to $7 \mathrm{ml} / \mathrm{min}$ infusions, the lowest $C_{\text {out }} / C_{\text {in }}$ was $0.12(E R=0.88)$, but values were as high as $0.45(E R=0.55)$, and in fact, only 3 of all 10 experiments supported galactose being a high-clearance drug $(E R>0.7)$ at the 6 to $7 \mathrm{ml} / \mathrm{min}$ infusion. The average $E R$ of all 10 experiments was $0.68 \pm$ 0.10 (S.D.). Of further concern with the Keiding and Chiarantini report is that when comparing clearance from the 10 to 11 to the 6 to $7 \mathrm{ml} / \mathrm{min}$ conditions, for 4 of the 10 experiments, clearance was higher at the lower blood flow of 6 to $7 \mathrm{ml} / \mathrm{min}$ (experiments 1, 2, 3, and 5). No model of hepatic elimination is consistent with this outcome. Perhaps these unacceptable results are a function of suboptimal experimental conditions, as authors mention that measured galactose concentrations were not corrected for perfusate volume changes due to evaporation, since they reason that the degree of evaporation in the recirculating system was approximately equal to the volume of galactose infusate $(22 \mathrm{ml})$. Furthermore, no studies were conducted to confirm that galactose elimination was in linear range at concentrations tested. Finally, based on the average rat liver weight of $6.87 \mathrm{~g}$, a flow rate of $6 \mathrm{ml} / \mathrm{min}$ corresponds to an average flow rate of $0.87 \mathrm{ml} / \mathrm{min}$ per gram, which is likely too low to fully perfuse the livers.

The second high-clearance compound investigated in IPRL studies is taurocholate. Smallwood et al. (1988) investigated taurocholate in the IPRL with changing fraction unbound in both recirculating and singlepass perfusion studies. In both experimental designs, the experimental data were fit equally well by the well-stirred and the dispersion models, and in fact, these two models could not be differentiated from one another (represented by the same line in their Fig. 2) because of very high average-fitted dispersion numbers of $5.0 \times 10^{7}$ (single-pass) and 
13.3 (recirculating). The authors acknowledge that when the dispersion number was sufficiently large (approaching infinity) the dispersion model approached the well-stirred model (infinite mixing) and that their observed dispersion numbers were "sufficiently large that the dispersion model has 'collapsed' into the venous equilibrium model extreme." Yet they conclude, "Moreover, perhaps it is time to relinquish the venous equilibration model, which, although operationally accurate, is conceptually flawed," which highlights the hesitation of the field to accept a model that best fits the data simply because of its limited physiologic relevance. A reviewer of this manuscript questions the trustworthiness of this report because the authors used total radioactivity as a measure of taurocholate (which forms a sulfate) and because experimental details related to the duration of these experiments were not clearly stated (although they can be deduced and appear to be a reasonable length of time). Additionally, rat liver weights were not reported, therefore it is not possible to assess the viability of the preparation for a continued high flow rate of $32 \mathrm{ml} / \mathrm{min}$, which typically should not exceed $3 \mathrm{ml} / \mathrm{min}$ per gram liver weight. However, reported rat liver weights from other IPRL studies cited here and in the literature range from 5.51 to $15.4 \mathrm{~g}$ for rats weighing 200-400 g. Therefore, a flow rate of $32 \mathrm{ml} / \mathrm{min}$ could be reasonably sustained for rat livers weighing approximately $10.5 \mathrm{~g}$, which is quite likely in this study given that the rats weighed between 250 and 300 g. Smallwood and coworkers (Ching et al., 1989) repeated their single-pass IPRL studies with taurocholate 1 year later and again found that in each of their six replicates, the well-stirred model best described taurocholate elimination since "dispersion number was greater than $10^{15}$ in all experiments and was therefore taken as infinite."

Roberts et al. (1990) also investigated taurocholate elimination in IPRL studies by varying both protein binding and flow. Figure 2 depicts experimental data from two different experiments from this publication in which protein binding was altered by experiments containing $0 \%$, $0.5 \%$, or $5 \%$ albumin at a flow rate of $10 \mathrm{ml} / \mathrm{min}$ versus the hypothetical well-stirred and dispersion models [two values were from the experiments designed to alter protein binding $(0.5 \% \mathrm{vs} .5 \%$ albumin $)$ at a flow of $10 \mathrm{ml} / \mathrm{min}$, and two values were from the $10 \mathrm{ml} / \mathrm{min}$ experiments designed to alter flow, which were run at both $0 \%$ and $5 \%$ albumin)]. From this figure, it is difficult to suggest a preference of one model versus another, especially given the large S.D.s for the potentially discriminating data points with the moderate protein-binding value. The authors comment on the inadequacy of the fit to the well-stirred model but make no direct comparison with the dispersion model until the final sentence of the manuscript, in which they excuse its insufficiency by

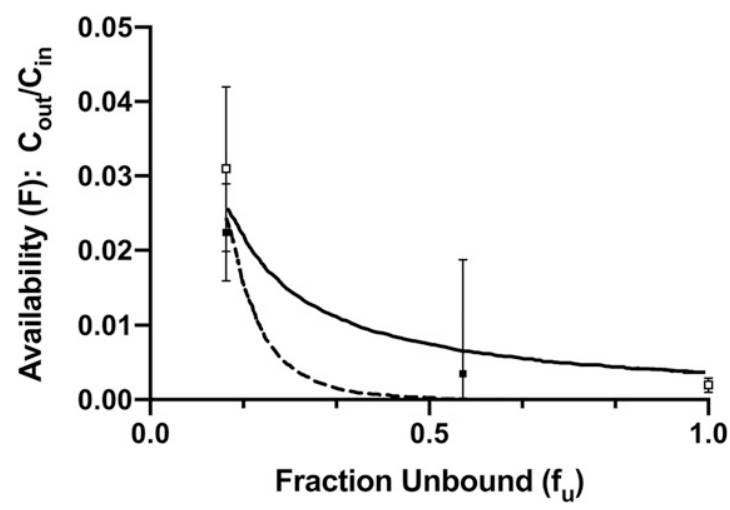

Fig. 2. $F$ predictions of well-stirred (upper solid line) and dispersion (lower dashed line) models of taurocholate availability with changes in fraction of taurocholate unbound in perfusate for two different experiments reported by Roberts et al. (1990). Observed hepatic availability values (mean \pm S.D.) are depicted for experiments containing $5 \%$ albumin $\left(f_{u}=0.14\right), 0.5 \%$ albumin $\left(f_{u}=0.56\right)$, or $0 \%$ albumin $\left(f_{u}=1.0\right)$ and are experimentally calculated by $\mathrm{C}_{\text {out }} / \mathrm{C}_{\mathrm{in}}$. indicating that "alterations in albumin content results in availabilities that require an albumin-mediated transport system to be used in conjunction with the dispersion model." Roberts et al. (1990) purport to show preference for the dispersion model over the well-stirred model when blood flow changes, although no model comparison figure is provided. The authors state, "The well-stirred model is unphysiological...if the well-stirred model were applied to the flow data in Table V, availabilities of 0.007 (observed $0.048 \pm 0.061$ S.D.) and 0.12 (observed $0.18 \pm 0.08$ S.D.) would be predicted from $0 \%$ to $5 \%$ albumin at $37 \mathrm{ml} / \mathrm{min}$ using $10 \mathrm{ml} / \mathrm{min}$ data. With the correction for volume changes, the predicted availabilities are 0.004 and 0.059 . Thus, the well-stirred model does not account for the data obtained in this study." The S.D.s listed above were not in the original text but have been added by us. The authors again did not directly evaluate the fit of experimental data with variable flow to the dispersion model as they did for the well-stirred model.

IPRL Studies of Diazepam and Diclofenac. There are three experimental IPRL clearance studies with the low hepatic clearance drug diazepam and one with diclofenac from the last century, in which the drug had been manipulated to be high-clearance in the absence of plasma proteins (Rowland et al., 1984; Ching et al., 1989; Diaz-Garcia et al., 1992; Hussein et al., 1993). One study demonstrated preference of diazepam for the parallel-tube model (Rowland et al., 1984), and the other three studies demonstrated preference of diazepam (Ching et al., 1989; Diaz-Garcia et al., 1992) and diclofenac (Hussein et al., 1993) for the axial dispersion model versus the well-stirred model. In the case of the Hussein et al. (1993) diclofenac study, the authors admit that "the improvement [of the dispersion model] over the well-stirred model was statistically significant in four of the eight preparations only," highlighting the variability associated with their results as well as that the reported success of the dispersion model was due to the fact that it was approximating the well-stirred model in half of their replicates. A similar degree of variability in model preference was also observed in the Ching et al. (1989) diazepam study, in which for six replicates that support the dispersion model, three were fit with dispersion numbers of approximately zero (the parallel-tube model), and one was fit with a dispersion number of infinity (the well-stirred model).

Furthermore, it should be highlighted that except for zero addition of protein to the perfusion solution, no other experimental results in these studies can adequately differentiate organ hepatic clearance models in all four of these publications. Additionally, the high degree of variability associated with the zero protein experiments for these highly proteinbound drugs is noteworthy. Wang and Benet (2019) very recently repeated these diazepam IRPL studies at zero protein concentration but also at very low albumin concentrations $(0.025 \%$ and $0.05 \%)$ with the intent of including more than a single model-discriminating experimental data point as well as potential mitigation of the variability associated with zero protein addition conditions. Results confirmed that at zero albumin concentration, data were consistent with the parallel-tube model as reported by Rowland et al. (1984). The results exhibited high variability, as also was seen in the previous Rowland laboratory studies. However, at $0.025 \%$ and $0.05 \%$ albumin, the results were preferentially consistent with the well-stirred model.

IVIVE Approaches. An alternate indirect methodologic approach to evaluate previously published IPRL data was proposed by Roberts and Rowland (1986) to support the dispersion model. In that analysis, in vitro measures of $C L_{i n t}$ were used after IVIVE techniques to predict in vivo $C L_{\text {int }}$ for drugs with published IPRL data. The predictions of in vivo $C L_{\text {int }}$ were further used to calculate an efficiency number $\left(R_{N}=f_{u}\right.$ - $C L_{\text {int }} / Q_{H}$ ) based on experimental conditions of $Q_{H}$ and $f_{u}$ from the IPRL studies, and these values were plotted against experimentally observed $F$ from the same IPRL studies. For 10 drugs, the predictive $R_{N}$ values were determined, and for high extraction ratio compounds 
(alprenolol, lidocaine, meperidine, phenacetin, and propranolol), the results appeared to be best described by the dispersion model (Fig. 3). This analysis was further presented subsequently by Iwatsubo et al. (1996) and included four additional drugs from the literature, which has been recreated in Fig. 3. The outcome for this indirect approach was unexpected since for three of the five high-clearance compounds included in this analysis, there are published IPRL experimental studies directly testing model preference, showing that the data only fit the wellstirred model: changing blood flow for lidocaine (Pang and Rowland, 1977; Ahmad et al., 1983) and meperidine (Ahmad et al., 1983) and changing protein binding for propranolol (Jones et al., 1984, 1985). How can this difference be explained? In contrast to Roberts and Rowland (1986), Iwatsubo et al. (1996) emphasize that the indirect IVIVE analysis is dependent on the assumption that in vitro determination of $C L_{\text {int }}$ will accurately predict in vivo $C L_{\text {int }}$ and $C L_{H}$. Iwatsubo et al. argued in 1996 that this was a valid assumption and that any difference between predicted and observed clearance was negligible. They provide the data available in 1996 to support this contention. But with time, it has been recognized by these authors that this assumption is incorrect (Chiba et al., 2009), and as Rowland and Pang (2018) note, "IVIVE tends to underpredict the estimated in vivo hepatic clearance (Hallifax et al., 2010) for poorly understood reasons." Bowman and Benet (2019) recently reported that of 19 drugs shown clinically in humans to be high $E R$, only $1(5.5 \%)$ of 18 from human hepatocyte $C L_{\text {int }}$ measurements and only $3(15.8 \%)$ of 19 from human microsomal $C L_{\text {int }}$ measurements were correctly predicted to be high $E R$. For studies of high in vivo $E R$ drugs in rats, only two $(22.2 \%)$ of nine were predicted to be high $E R$ by rat hepatocytes and only two (25\%) of eight were predicted to be high $E R$ by rat microsomes, supporting observations by the field that the IVIVE underprediction is not a species-specific phenomenon. It is obvious today that in vitro $C L_{i n t}$ markedly under-predicts in vivo $C L_{i n t}$, and the Roberts and Rowland (1986) and Iwatsubo et al. (1996) analyses today might have been very different than those published last century. Therefore, an IVIVE-based approach to model discrimination cannot reliably be trusted without consideration of the degree of underprediction expected for the drugs studied.

Vascular Dispersion and Axial Tissue Diffusion. Rivory et al. (1992) attempted to explain "the paradoxical ability of the venousequilibration model to describe the steady-state kinetics of lipophilic drugs such as lidocaine, meperidine and propranolol" versus more physiologic relevant models. The authors attempt to validate the complex but physiologically relevant tissue-diffusion model in a 43page paper with 35 equations and propose that "vascular dispersion is of major importance to the availability of poorly diffusible compounds, whereas axial tissue diffusion becomes increasingly dominant for highly diffusive and partitioned substances." Reanalysis of a number of the above IPRL experiments (Pang and Rowland, 1977; Ahmad et al., 1983; Jones et al., 1984) resulted in figures that essentially demonstrate that the tissue-diffusion model can also accommodate the data by approximating the well-stirred model fits.

\section{Discussion}

Although alternate models to the well-stirred model for hepatic drug elimination have been examined since 1977, we find no comprehensive review of the concordance of these models with experimental IPRL results. The evaluation of high-ER compounds in model discrimination is critical, as each hepatic disposition model diverges from one another for high-clearance compounds. Surprisingly, there are only four IPRL studies that have directly evaluated these models for high- $E R$ drugs (Pang and Rowland, 1977; Ahmad et al., 1983; Jones et al., 1984, 1985). In all four of these studies, data are preferentially consistent with the

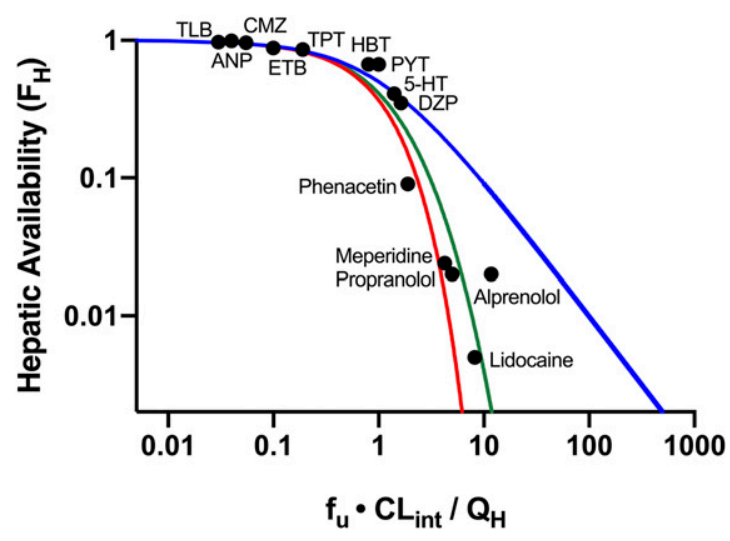

Fig. 3. Plots of $F_{H}$ vs. $f_{u} \cdot C L_{i n t} / Q_{H}$, including the theoretical well-stirred, paralleltube, and dispersion model relationships based on data from Roberts and Rowland (1986) and Iwatsubo et al. (1996). Data points assuming no error in IVIVE prediction are depicted. The five high extraction ratio compounds included in this analysis (alprenolol, lidocaine, meperidine, phenacetin, and propranolol) are labeled in the figure. Additional compounds (low and moderate extraction ratio) are labeled with the following abbreviations: ANP, antipyrine; CMZ, carbamazepine; DZP, diazepam; ETB, ethoxybenzamide; HBT, hexobarbitone; 5-HT, 5-hydroxytryptamine; PYT, phenytoin; TLB, tolbutamide; TPT, thiopental. Blue, green, and red lines depict the well-stirred, dispersion, and parallel-tube model relationships.

well-stirred model, a fact acknowledged by the authors in each publication.

High-clearance nondrug substrates have also been evaluated in IPRL studies for galactose and taurocholate. The frequently cited Keiding and Chiarantini (1978) galactose IPRL study concluded that the parallel-tube model is preferentially consistent with the experimental data; however, critical examination of their experimental results calls their conclusion into question. Although galactose is known to be a high-clearance compound (Goresky et al., 1973; Henderson et al., 1982), galactose did not have a high $E R$ in any experiment run at 10 to $11 \mathrm{ml} / \mathrm{min}$ (with an average $E R$ of $0.46 \pm 0.11$ ). At the 6 to $7 \mathrm{ml} / \mathrm{min}$ infusions, galactose was only observed to be a high- $E R$ compound in 3 of 10 replicates. But of utmost concern was that clearance was observed to increase as flow was decreased from 10 to $11 \mathrm{ml} / \mathrm{ml}$ to 6 to $7 \mathrm{ml} / \mathrm{min}$ in 4 of 10 experiments. This outcome violates hepatic physiology, and no model of hepatic disposition is consistent with this outcome. The validity of the Keiding and Chiarantini (1978) publication in support of the paralleltube model is highly questionable, and these data should not be further cited in the literature as supporting an alternate model of hepatic elimination.

A second high-clearance nondrug substance, taurocholate, was investigated by Smallwood et al. (1998) under conditions of altered protein binding. In both single-pass and recirculating perfusion studies, their dispersion model fits had collapsed into the well-stirred model with very high average-fitted dispersion numbers $\left[5.0 \times 10^{7}\right.$ (single-pass) and 13.3 (recirculating)], as evidenced by both models being represented by the same line in their Figure 2. . Although they acknowledge that when dispersion number approaches infinity, the dispersion model simply approximates the well-stirred model, they hesitate to accept the conclusion that the well-stirred model is adequate because of its limited physiologic relevance. Ching et al. (1989) repeated the taurocholate single-pass IPRL studies, again finding that the well-stirred model best fit the observed data. Taurocholate was also investigated by Roberts et al. (1990) in IPRL experiments that varied protein binding and flow; resulting data were plagued with high variability precluding the ability to conclude model preference. Their experimental data from studies that altered protein binding are depicted in Fig. 2, and clearly no conclusion can be drawn given the huge variability associated with the model-discriminating 
protein-binding measurements. No figure is presented for experiments that altered flow. Although authors consistently describe the inadequacy of the well-stirred model fits, no direct comparisons nor statistical analyses are provided regarding the dispersion model fits.

Four studies were identified in which low-ER drugs were manipulated to be high-clearance in the absence of plasma proteins; one study indicates preference of diazepam for the parallel-tube model (Rowland et al., 1984) and the other three indicate preference of diazepam (Ching et al., 1989; Diaz-Garcia et al., 1992) and diclofenac (Hussein et al., 1993) for the dispersion model. The conclusions of these studies hinged on the experimental measurements conducted without plasma proteins that were plagued with a high degree of variability, resulting in inconsistencies in model preference for each replicate. For instance, the diclofenac study (Hussein et al., 1993) reported that the success of the dispersion model over the well-stirred model was only statistically significant in four of eight experiments, highlighting that the purported success of the dispersion model was simply due to its approximation of the well-stirred model in half of their replicates. Significant variability in model preference was also observed in the Ching et al. (1989) diazepam study; for six experiments reported to prefer the dispersion model, three were fit with dispersion numbers of zero (parallel-tube model), and one was fit with a dispersion number of infinity (the well-stirred model). In all these studies, the only model-discriminating conditions were those with zero protein in the perfusion media, and the high degree of variability associated with this condition is particularly noteworthy. For these reasons, we repeated these diazepam IPRL studies with additional low-albumin concentrations $(0.025 \%$ and $0.05 \%)$ to include more than a single model-discriminating experimental data point (Wang and Benet, 2019). These results confirmed the high degree of variability associated with the experimental measurement with zero protein in the perfusate. However, for the two additional very low albumin concentrations, results were preferentially consistent with the well-stirred model. Recent studies in the Poulin, Sugiyama, and Benet laboratories with hepatocytes report markedly improved IVIVE predictability in the presence of albumin than in its absence (Bowman and Benet, 2018; Poulin and Haddad, 2018; Kim et al., 2019). This could also explain the differences seen by Roberts et al. (1990) with zero protein concentration for taurocholate. Therefore, although our diazepam IPRL results (in the absence of protein in the perfusion media) support the high variability and outcomes observed by Rowland and coworkers, such results cannot be reasonably interpreted as supporting preference for any model.

Previous indirect model-discrimination approaches presented by Roberts and Rowland (1986) and Iwatsubo et al. (1996) are reproduced in Fig. 3 but are dependent on the assumption that IVIVE of hepatic clearance is accurate. Based on the contemporary understanding that in vitro measures of drug metabolism inexplicably and significantly underpredict in vivo drug clearance (Wood et al., 2017), it is reasonable that the IVIVE-based predictions of in vivo $C L_{\text {int }}$ used in determination of $R_{N}$ ( $x$-axis) are underpredictions. Therefore, it is expected that if it were possible to account for IVIVE underprediction of $C L_{\text {int }}$, the data points would shift rightwards toward the well-stirred model relationship.

Thus, in response to the title of this manuscript, we find no experimental data that reasonably or unambiguously support preference for the dispersion or parallel-tube models versus the well-stirred model of organ elimination when only entering and exiting drug concentrations are available, except for the studies of highly bound diazepam and diclofenac only at zero protein concentration. However, there are data that unambiguously show that $C_{\text {out }} / C_{\text {in }}$ measurements with changing blood flow and protein binding can only be fit by the well-stirred model. This outcome is unexpected if eq. 1 is assumed to be model-independent because it would be expected that data would sometimes support the well-stirred model (infinite mixing), sometimes the parallel-tube model (zero mixing), and sometimes neither of these models. We propose a simple reason why success is not consistent for more "physiological" hepatic models (compared with the well-stirred model) based on our contention of the model dependence of eq. 1 . That is, experimental data for steady-state IPRL studies for high-clearance drugs are consistent with eq. 1 , the well-stirred model relationship. When alternate hepatic disposition models approximate the boundary condition of the wellstirred model, successful fitting of the data is observed. Other explanations previously proposed to support alternate methodologies and models are flawed in assuming IVIVE is accurate and that perfusion studies in the absence of albumin yield exaggerated outcomes compared with even the smallest presence of protein. In our recent studies of diazepam, we confirmed the high variability associated with zero protein addition, however, because at two additional model-differentiating lowalbumin concentrations, the data were best described by the well-stirred model (Wang and Benet, 2019).

It is difficult to understand why the four IPRL studies that directly test model preference for highly cleared drugs that support the well-stirred model are undervalued by the field. We believe that the investigators were influenced by the unphysiologic aspects of the well-stirred model, by assumptions made last century that are no longer valid today, and by lack of critical review of previously reported studies, resulting in inappropriate interpretation of the available experimental data. We emphasize the frequently cited quote of 1965 Nobel Prize physicist Richard Feynman(Feynman, 1965): "It doesn't matter how beautiful your theory is, it doesn't matter how smart you are. If it doesn't agree with experiment, it's wrong." We agree that the dispersion model is more physiologic than the well-stirred model and believe that it is more beautiful. We know that it is impossible for the well-stirred model to capture the complexities of liver physiology, including heterogeneity in enzymatic expression and dispersive flow throughout the liver. But, when experimental studies are limited to measurements for the entering and exiting drug concentrations of the elimination organ at steady state and eq. 1, only the well-stirred model analysis is possible. The results summarized here do not indicate that the well-stirred model is an accurate representation of true hepatic elimination, it simply highlights that the well-stirred model is the best we can do when eq. 1 is used to calculate clearance. With recent advancement of experimental and analytical techniques that can allow us to measure dynamic intracellular hepatic concentrations and with respect to time as well as location within the organ, there is significant potential for our field to drastically improve the current oversimplified models of organ disposition.

\section{Authorship Contributions}

Participated in research design: Sodhi, Wang, Benet. Performed data analysis: Sodhi, Wang, Benet.

Wrote or contributed to the writing of the manuscript: Sodhi, Wang, Benet.

\section{References}

Ahmad AB, Bennett PN, and Rowland M (1983) Models of hepatic drug clearance: discrimination between the 'well stirred' and 'parallel-tube' models. J Pharm Pharmacol 35:219-224.

Benet LZ, Liu S, and Wolfe AR (2018) The universally unrecognized assumption in predicting drug clearance and organ extraction ratio. Clin Pharmacol Ther 103:521-525.

Bowman CM and Benet LZ (2018) An examination of protein binding and protein-facilitated uptake relating to in vitro-in vivo extrapolation. Eur J Pharm Sci 123:502-514.

Bowman CM and Benet LZ (2019) In vitro-in vivo extrapolation and hepatic clearance-dependent underprediction. J Pharm Sci 108:2500-2504.

Chiba M, Ishii Y, and Sugiyama Y (2009) Prediction of hepatic clearance in human from in vitro data for successful drug development. AAPS J 11:262-276.

Ching MS, Morgan DJ, and Smallwood RA (1989) Models of hepatic elimination: implications from studies of the simultaneous elimination of taurocholate and diazepam by isolated rat liver under varying conditions of binding. J Pharmacol Exp Ther 250:1048-1054.

Cobelli C, Carson ER, Finkelstein L, and Leaning MS (1984) Validation of simple and complex models in physiology and medicine. Am J Physiol 246:R259-R266.

Díaz-García JM, Evans AM, and Rowland M (1992) Application of the axial dispersion model of hepatic drug elimination to the kinetics of diazepam in the isolated perfused rat liver. J Pharmacokinet Biopharm 20:171-193. 
Feynman RP (1965) The Character of Physical Law p 156, The M.I.T. Press, Cambridge, MA and London.

Goresky CA, Bach GG, and Nadeau BE (1973) On the uptake of materials by the intact liver. The transport and net removal of galactose. J Clin Invest 52:991-1009.

Hallifax D, Foster JA, and Houston JB (2010) Prediction of human metabolic clearance from in vitro systems: retrospective analysis and prospective view. Pharm Res 27:2150-2161.

Henderson JM, Kutner MH, and Bain RP (1982) First-order clearance of plasma galactose: the effect of liver disease. Gastroenterology 83:1090-1096.

Hussein Z, Evans AM, and Rowland M (1993) Physiologic models of hepatic drug clearance: influence of altered protein binding on the elimination of diclofenac in the isolated perfused rat liver. J Pharm Sci 82:880-885.

Iwatsubo T, Hirota N, Ooie T, Suzuki H, and Sugiyama Y (1996) Prediction of in vivo drug disposition from in vitro data based on physiological pharmacokinetics. Biopharm Drug Dispos 17:273-310.

Jones DB, Ching MS, Smallwood RA, and Morgan DJ (1985) A carrier-protein receptor is not a prerequisite for avid hepatic elimination of highly bound compounds: a study of propranolol elimination by the isolated perfused rat liver. Hepatology 5:590-593.

Jones DB, Morgan DJ, Mihaly GW, Webster LK, and Smallwood RA (1984) Discrimination between the venous equilibrium and sinusoidal models of hepatic drug elimination in the isolated perfused rat liver by perturbation of propranolol protein binding. J Pharmacol Exp Ther 229:522-526.

Keiding S and Chiarantini E (1978) Effect of sinusoidal perfusion on galactose elimination kinetics in perfused rat liver. J Pharmacol Exp Ther 205:465-470.

Kim SJ, Lee KR, Miyauchi S, and Sugiyama Y (2019) Extrapolation of in vivo hepatic clearance from in vitro uptake clearance by suspended human hepatocytes for anionic drugs with high binding to human albumin: improvement of in vitro-to-in vivo extrapolation by considering the "albumin-mediated" hepatic uptake mechanism on the basis of the "facilitated-dissociation model". Drug Metab Dispos 47:94-103.

Pang KS and Rowland M (1977) Hepatic clearance of drugs. II. Experimental evidence for acceptance of the "well-stirred" model over the "parallel tube" model using lidocaine in the perfused rat liver in situ preparation. J Pharmacokinet Biopharm 5:655-680.

Poulin P and Haddad S (2018) Extrapolation of the hepatic clearance of drugs in the absence of albumin in vitro to that in the presence of albumin in vivo: comparative assessement of 2 extrapolation models based on the albumin-mediated hepatic uptake theory and limitations and mechanistic insights. J Pharm Sci 107:1791-1797.
Rivory LP, Roberts MS, and Pond SM (1992) Axial tissue diffusion can account for the disparity between current models of hepatic elimination for lipophilic drugs. J Pharmacokinet Biopharm 20:19-61.

Roberts MS, Fraser S, Wagner A, and McLeod L (1990) Residence time distributions of solutes in the perfused rat liver using a dispersion model of hepatic elimination: 1. Effect of changes in perfusate flow and albumin concentration on sucrose and taurocholate. J Pharmacokinet Biopharm 18:209-234.

Roberts MS and Rowland M (1986) Correlation between in-vitro microsomal enzyme activity and whole organ hepatic elimination kinetics: analysis with a dispersion model. J Pharm Pharmacol 38: $177-181$

Rowland M (1972) Influence of route of administration on drug availability. J Pharm Sci 61:70-74. Rowland M, Leitch D, Fleming G, and Smith B (1984) Protein binding and hepatic clearance: discrimination between models of hepatic clearance with diazepam, a drug of high intrinsic clearance, in the isolated perfused rat liver preparation. J Pharmacokinet Biopharm 12:129-147.

Rowland M and Pang KS (2018) Commentary on "The universally unrecognized assumption in predicting drug clearance and organ extraction ratio". Clin Pharmacol Ther 103:386-388.

Smallwood RH, Morgan DJ, Mihaly GW, Jones DB, and Smallwood RA (1988) Effect of plasma protein binding on elimination of taurocholate by isolated perfused rat liver: comparison of venous equilibrium, undistributed and distributed sinusoidal, and dispersion models. J Pharmacokinet Biopharm 16:377-396.

Wang H-J and Benet LZ (2019) Protein binding and hepatic clearance: re-examining the discrimination between models of hepatic clearance with diazepam in the isolated perfused rat liver preparation. Drug Metab Dispos 47:1397-1402.

Wood FL, Houston JB, and Hallifax D (2017) Clearance prediction methodology needs fundamental improvement: trends common to rat and human hepatocytes/microsomes and implications for experimental methodology. Drug Metab Dispos 45:1178-1188.

Address correspondence to: Leslie Z. Benet, Department of Bioengineering and Therapeutic Sciences, Schools of Pharmacy and Medicine, University of California San Francisco, 533 Parnassus Ave., Rm U68, UCSF Box 0912, San Francisco, CA 94143. E-mail: leslie.benet@ucsf.edu 